\title{
MARKETING DIGITAL EN LAS PYMES DIGITAL MARKETING IN SMES
}

\author{
Autor 1. Camacho Rodriguez, Luz Dary \\ Escuela de Ciencias Administrativas, Contables, Económicas y de Negocios \\ Universidad Nacional Abierta y a Distancia UNAD, Bogotá
}

\section{RESUMEN}

Dado el impacto que tienen las pymes en la economía no se puede ser ajenas a los cambios que ha traído la revolución tecnológica en la manera de dinamizar el comercio. En este sentido, desde la academia se debe validar las necesidades de formación que se requieren para que éstas puedan ser competitivas en los nuevos retos que exige el mercadeo desde el contexto digital. Bajo esta perspectiva se ha llevado a cabo un ejercicio de indagación para reconocer el estado de conocimientos que tienen las pequeñas y medianas empresas en cuanto a las diferentes estrategias de mercadeo 3.0, pretendiendo identificar oportunidades de formación.

Palabras claves: marketing digital, pymes, competitividad.

\section{ABSTRACT}

Given the impact that SMEs have on the economy, they cannot be oblivious to the changes that the technological revolution has brought in the way to boost trade. In this sense, the academy must validate the training needs that are required so that they can be competitive in the new challenges that marketing demands from the digital context. Under this perspective, an inquiry exercise has been carried out to recognize the state of knowledge that small and medium-sized companies have regarding different marketing strategies 3.0, aiming to identify training opportunities.

Keywords: digital marketing, SMEs, competitiveness.

\section{INTRODUCCIÓN}

Pensar en todas las posibilidades que ofrece los avances tecnológicos en la gestión del área comercial de una empresa y las oportunidades de competición, hace que el marketing digital se convierta en un escenario interesante de investigación. Así indagar sobre qué tanto conocen las pymes acerca de este el tema es el punto de partida para la investigación. Indagación que se hizo con pequeños empresarios como grupos focales que dieron respuesta a interrogantes como ¿Conocen sobre el tema? ¿Diferencian las herramientas y estrategias del marketing digital? ¿Aplican el marketing digital en las empresas?

Los datos que arrojó el estudio realizado por el Ministerios de la Tecnologías de la Información y las Comunicaciones del Colombia (2018) revelan el impacto que ha traído los avances tecnológicos en la gestión comercial en la que han ido incorporando estrategias de comercialización del mercado análogo al mercadeo digital. Algunos resultados del estudio indican que:

- $75 \%$ de la mipymes a 2015 cuentan con conexión a internet

- Solo un $20 \%$ usan las conexiones a internet en el área comercial vs un $31 \%$ a la administración de la empresa

- El $36 \%$ de las empresas cuenta con un sitio web 
- Manifiestan necesidades de un $46 \%$ para el uso de web, $45 \%$ para el uso de redes sociales, $45 \%$ para uso de programa ofimática,35\% para la elaboración de contenidos digitales y un $35 \%$ para uso de herramientas colaborativas

- Solo el $38 \%$ de las empresas tienen presencia en redes sociales

Esta red de redes ha hecho posible el concepto de globalización, con lo cual se ha derribado fronteras geográficas sin salir de casa. Según el observatorio de la Cámara de Comercio Electrónico (2018) mas de 87 millones de transacciones en línea realizaron los colombianos en el 2017 cifra que representa 51,2 billones de pesos.

Estos resultados reflejan el impacto que conlleva aprender a moverse en el mercadeo digital, mas que una oportunidad, un reto para las pymes el aprender a desenvolverse en este contexto.

De allí que los resultados que arrojó la investigación será una contribución a la identificación de las necesidades de formación para los empresarios y propuestas a los contenidos curriculares de los programas de formación en el campo del mercadeo.

A continuación, se presenta un recorrido por la evolución del marketing digital con miras a ubicar al lector para entender el instrumento de recopilación de datos a través de los cuales se compruebe los saberes de los empresarios acerca de este tema.

\section{Revisión bibliográfica}

Por si solo el marketing se ha integrado a la dinámica propia de las organizaciones, dada su influencia en el alcance de objetivos económicos y del posicionamiento de la empresa y de sus productos en el mercado. En algunas empresas es un área claramente definida y hasta la columna vertebral, aunque, en otras de manera inconsciente, rudimentaria o avanzada han ido valorando el valor que genera para sus clientes esta disciplina en la organización, no solo como un área estratégica sino también por su operatividad en la búsqueda de relaciones de intercambio entre la empresa y su entorno, con miras a la satisfacción de las necesidades y deseos del mercado mediante la identificación, creación, desarrollo y cubrimiento de la demanda para generar valor y satisfacción.

La evolución que ha sufrido el marketing ha ido de la mano con los cambios sociales, económicos y políticos. En este sentido, la revolución tecnológica y de información se ha extendido a la gestión del mercadeo, que mas que tratarse de cambiar lo tradicional o analógico a un mundo virtual, ha dado paso a nuevos conceptos, términos y herramientas que se han vuelto una realidad.

No cabe duda porque la comunicación ha sufrido evoluciones significativas, que ha hecho posible la conexión con cualquier persona sin salir de casa, rompiéndose paradigmas de espacio y tiempo. Al mismo tiempo, cambios sustanciales en los hábitos, la manera de comprar y la forma de interacción entre la empresa y el consumidor.

En este punto, las empresas las empresas para ser competitivas, deben incorporar herramientas tecnológicas para mejor su gestión de marketing. El éxito en esta nueva era está marcado por el valor creado para sus clientes, colaboradores y accionistas, a la creación de experiencias de consumo memorable, a seguidores de marcas, a una conversación permanente con el cliente, donde coproducen el producto o el servicio.

Giraldo (2016, p.261) define el marketing digital como "la implementación de un conjunto de herramientas, tácticas y estrategias comerciales dentro del entorno digital que incluyan todos los espacios relevantes donde el usuario interactúe con la marca o empresa", de igual manera, expresa que los medios digitales se caracterizan por la inmediatez, la interactividad, la colaboración, el intercambio, el compartir información, la creación de comunidades, la transparencia, la medición en el tiempo real de la estrategia. 
Los avances tecnológicos han sido un aliado para el marketing digital, entendida como la aplicación de las estrategias de comercialización llevadas a cabo en los medios digitales (gracias a revoluciones tecnológicas de hardwards y software) a través de las cuales se han facilitado el acercamiento para enganchar a sus clientes en mayor cantidad, efectividad y potencialidad de ventas, que invita a ser mas eficientes, amigables y accesibles para satisfacer los clientes actuales que desean acceder de forma mas rápida y continua con la información de la empresa.

En efecto, la revolución tecnológica ha influido en el comportamiento de los consumidores, y por la dinámica propia del marketing. Kotler, Kartajaya \& Setiwan (2011) señala en su libro Marketing 3.0 las tres grandes evoluciones que ha sufrido. En primera instancia, el marketing 1.0, enfocado al producto, posteriormente la evolución hacia el marketing 2.0, cuyo enfoque se centra en el consumidor. El marketing 3.0 adquiere una perspectiva centrada en el interés para la humanidad. Hoy en día, y con el marketing 4.0, la perspectiva se centra en la inteligencia artificial. Los detalles de cada una de estas etapas se pueden describir de la siguiente manera:

- Con el marketing 1.0, la comunicación entre empresa - cliente fluye en una sola dirección con contenido estáticos y controlados directamente por los creados o gestores webs, implicando a la organización programadores responsables del proceso que implicaba inversión alta de recursos.

- Para el marketing 2.0 la comunicación se dinamiza un cambio radical con la incorporación de nuevas herramientas como blogs, redes sociales. En esta etapa se crea y comparte conocimientos, experiencias y se añaden contenidos, dejando de ser el cliente desconocido y pasivo, creándose una gigantesca plataforma tecnológica y de servicios, tales como youtube, Facebook, bloooger, twitter, linkedln, Messenger, Skype, entre otras.

- En el marketing 3.0 se cuenta con un enfoque con base social, ético y medioambiental cuyas herramientas tecnológicas facilitan la conexión con los clientes. Es la revolución de mass media (masificación de los medios de comunicación) y el comercio electrónico. Y finalmente, está surgiendo la web 4.0 que, a partir de la inteligencia artificial, las personas como objetivos se comunicarán entre sí con inteligencia compartida se desarrolla aplicaciones tales como database, wedgest, social media sharing, social networking.

De igual manera, la tecnología ha generado cambios de actitudes en los consumidores. Dejaron de ser entes pasivos a pasaron a ser pro consumidores (consumen contenidos y generan contenidos). Ya no toman decisiones desde el desconocimiento, sino que están bien informados, convirtiéndose en una fuente activa de información para las empresas.

Hoy en día se identifican consumidores les gusta demostrar su capacidad de generar valor ante los demas, unos quieren recibir productos o servicios personalizados adaptándolos a su estilo de vida particular, otros persiguen incentivos económicos que ofrezca algunas empresas por sus esfuerzos de co- creación, que para algunos es una oportunidad para asegurar un empleo y otros lo hacen por solo diversión.

Fernández (2013) señala que cada vez las comunicaciones digitales han tomado un lugar preponderante dentro de la vida cotidiana. La toma de decisiones hoy en día se basa en información que se encuentra en motores de busca o las referencias que reciben de amigos 


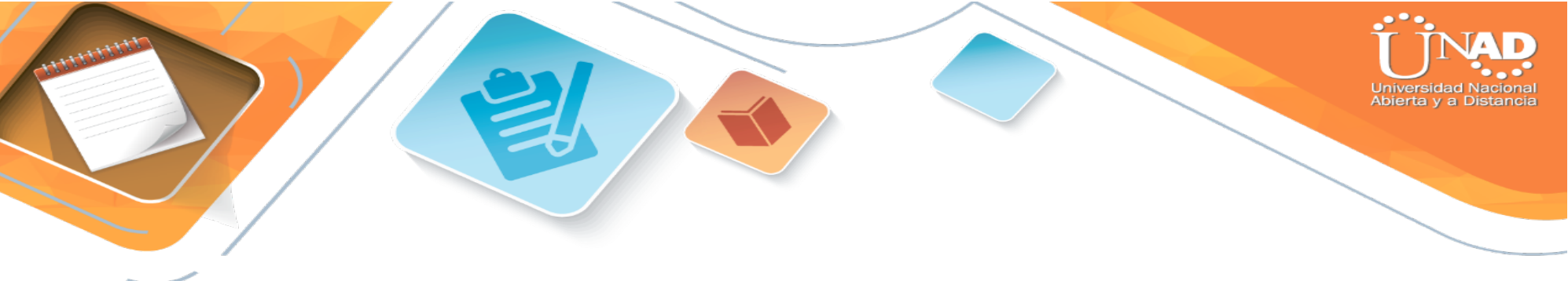

o desconocidos acerca de los productos que compran (comentarios). Las cifras así lo indican. De acuerdo con Smdigital (2014):

- 3 billones de personas en el mundo ya están conectadas a internet, aproximadamente la mitad de la población mundial. Para el caso colombiano aproximadamente 21 millones de usuario.

- 4 billones de usuarios de telefonía móvil, de los cuales el $25 \%$ con Smartphone. El teléfono se consolida como el medio rey por su nivel de penetración.

- Aumento en el tiempo consumido de consulta

- El comercio electrónico supera los 1.2 trillones de dólares para compras por internet

El manifiesto Cluetrain (Levine, Locke, Searls \& Weinberger, 2009) presenta 95 hipótesis sobre el comportamiento actual del mercado, que reflejan la realidad actual, obligando a tener una visión del cliente que puede acceder a información sobre la empresa y el producto. Entre ella se encuentra:

- Los mercados consisten en seres humano, no de sectores demográficos

- La internet hace posible tener conversaciones entre seres humanos que simplemente eran imposibles en la era de los medios de comunicación masiva.

- No hay secretos. El mercado en red sabe mas que las empresas acerca de sus propios productos. Y ya sea que las noticias sean buenas o malas, se las comunican a todo el mundo.

- Las compañías que no se dan cuenta que sus mercados ahora están interconectados persona-a-persona, y por consecuencia volviéndose mas inteligentes y profundamente unidos en conversación, están perdiendo su mejor oportunidad.

- Las empresas ahora pueden comunicarse con sus mercados directamente. Esta podría ser su última oportunidad si la desperdician.

Esa nueva realidad virtual, aunque suene contradictorio, obliga a actuar proactivamente a las empresas, dejando de lado la era del "marketing obsolet" como lo ha denominado Kotler (1999) a una era donde el marketing debe ajustarse al marketing de valor, aprovechando los beneficios y ventajas que proporciona las TICs, incorporado nuevas estrategias que impulsan la productividad y el crecimiento de las empresas

En este nuevo contexto virtual, el marketing digital ha ido ganando terreno, surgiendo términos como del marketing on line $y$, emaillings, social media, buzzmarketing, blog markering, marketing viral, herramientas como el poscast, blogs, wikis, widget, redes sociales, comunidades, agregados y video on line, redes sociales y comunidades, pago por click, adwords, marketing de buscadores etc., el marketing de contenidos. Internet se ha incorporado a la vida diaria ya no solo para la búsqueda de información, sino como medio de entretenimiento y como medio de comercialización.

De las cuatro Ps (producto, precio, plaza, promoción) del marketing análogo se han incorporado al mundo del marketing digital cinco variables: posicionamiento en los 



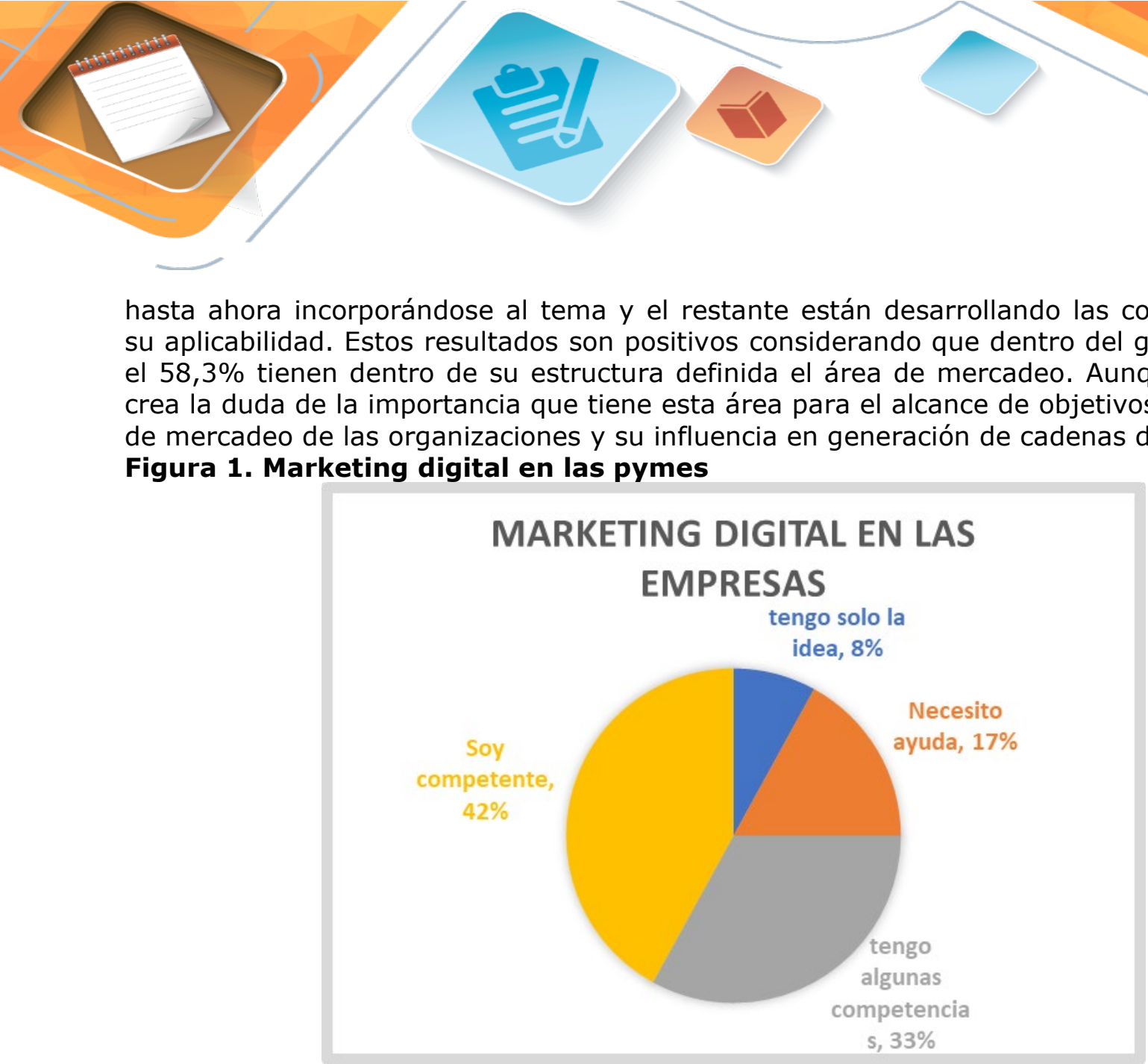

\section{Fuente: elaboración propia}

En la figura 2, un $75 \%$ (sumando soy competente y tengo algunas competencias) de empresarios incorporan dentro de sus estrategias comerciales el uso de las páginas web como canal de comunicación y de captación de clientes, debido a que realizan análisis sobre su efectividad y pertinencia, no obstante, solo un 33\% aprovechan esta información en pro de la identificación de palabras keyword o palabras claves que dinamicen el tráfico hacia la web de la página y se evite los motores de búsqueda de pago (SEM), ademas del análisis de patrones y comportamientos de compra para el diseño de estrategias de comunicación y la dinamización a compras efectivas. 


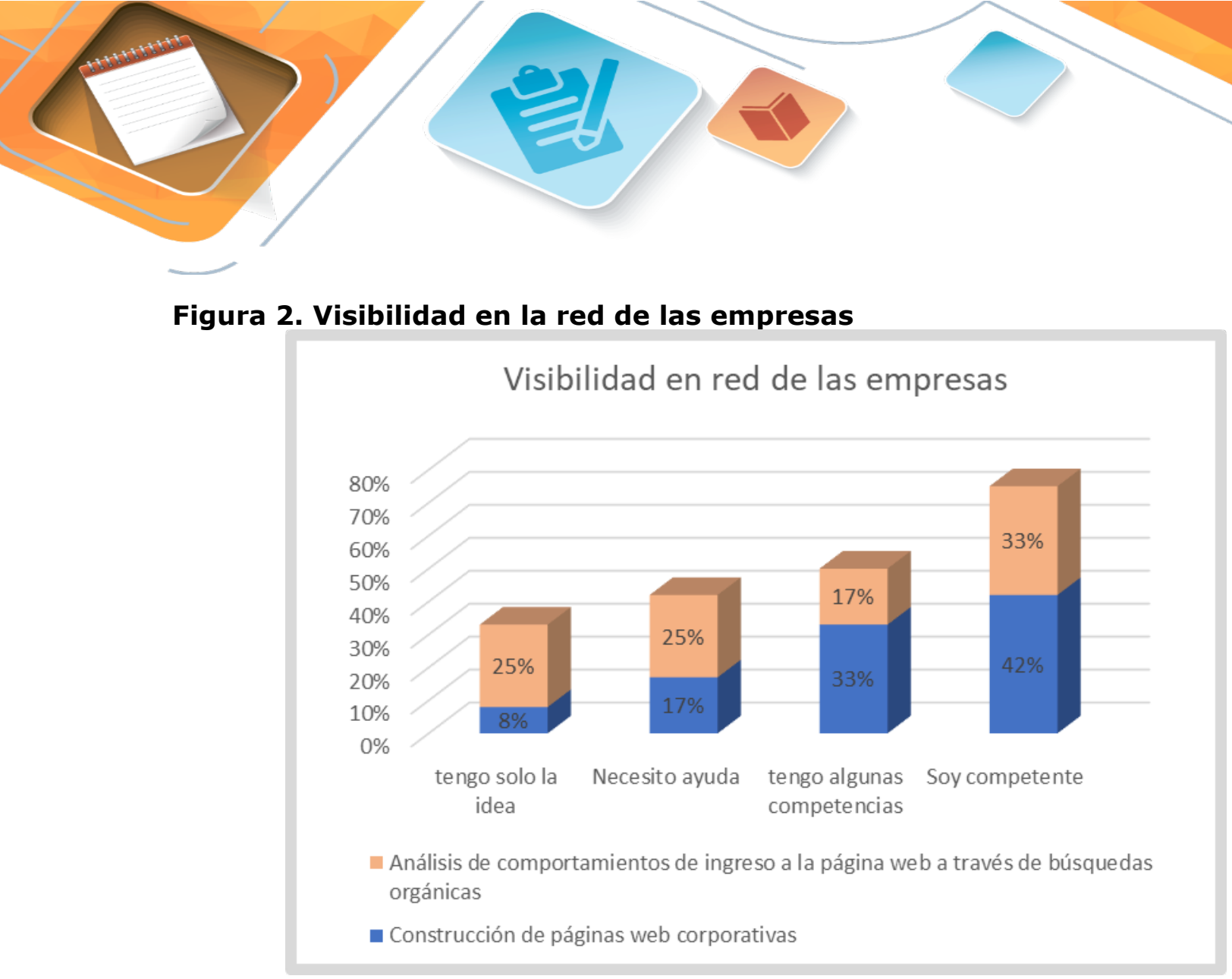

\section{Fuente: elaboración propia}

Retomando la evolución del marketing que describe Kotler, Kartajaya \& Setiwan (2011), el $17 \%$ de los empresarios están hasta ahora pasando por la incorporación de estrategias de marketing 1.0; el 66\% manejan y usan herramientas del marketing de 2.0 donde la redes como Facebook y, el WhatsApp son las de mayor difusión. Mientras que el $61 \%$ de los empresarios están dando su primer pino para la incorporando las estrategias del marketing 3.0, dado que se evidencia debilidades aún en la construcción de big data claves para la caracterización de los clientes, sus procesos de compra, necesidades y expectativas. Información insumo para la construcción de estrategias comerciales que promuevan la competitividad en el mercado de las organizaciones.

Uno de los retos del marketing 3.0 es el uso de tecnologías que faciliten la conexión con los clientes, la revolución de mass media y el comercio electrónico. Como lo indica Giraldo (2016) en la implementación del marketing digital la meta es hacer uso de herramientas que promuevan la interacción con entre los clientes y empresas. En la figura 3 se presentan los resultados donde se resalta el dominio del correo electrónico donde el comercio electrónico no está a la par, que ratifica la masificación del correo sin evaluar su efectividad.

Frente al caso de la conexión con los clientes, aun los microempresarios tienen camino por recorrer, dado que herramientas como webinars, timeline, pots solo son usadas por el $17 \%$ y un $8 \%$ hace uso del Zoom. Que solo el $33 \%$ realice inteligencia comercial implica retos en la implementación de 


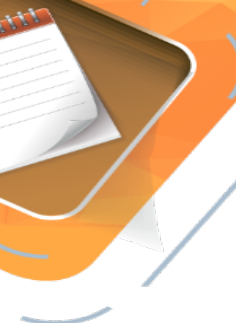

herramientas que consoliden información para la toma de decisiones comerciales, donde se presume el uso de fuentes tradicionales. Uno de los beneficios de las TICs (tecnologías de la información y la comunicación) para las empresas es el poder para conocer los comportamiento y patrones de consumo para ofrecer productos con valor agregado que se adaptan a su estilo de vida.

Figura 3. Conocimientos marketing $\mathbf{3 . 0}$ de los empresarios

\section{Conocimientos de marketing 3.0}

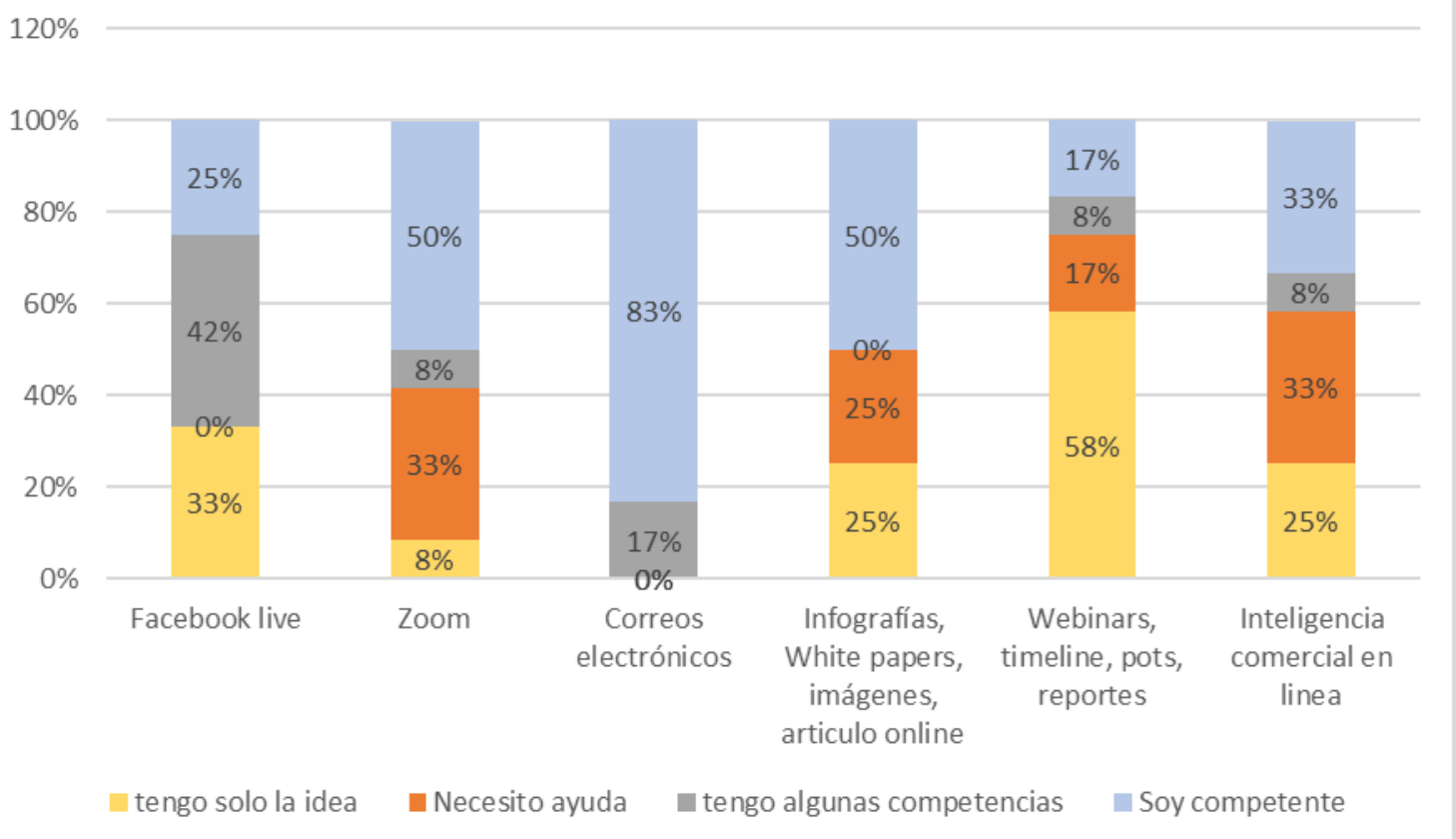

\section{Fuente: elaboración propia}

En gran medida las posibilidades de expresión se incrementan en los medios sociales, los consumidores podrán influir cada vez con otros consumidores mediante sus opiniones y experiencias, dedicando tiempo para revisar información previa en buscadores de las empresas, sus productos o servicios, antes de tomar la decisión de compra. En este sentido en la investigación se identifica que aproximadamente el $50 \%$ de empresarios han incorporado la construcción de contenidos y la participación en redes sociales en sus estrategias comerciales. Siendo el Facebook la red social mas usada (89\%), aclarando que solo un $25 \%$ está usando Facebook Live $^{2}$. Se ratifica como las comunicaciones digitales están tomando un lugar preponderante de la vida cotidiana (Fernández, 2013).

La inmersión en el marketing digital de las pequeñas y medianas empresarias está siendo real. Sin embargo, su masificación y cambios constantes hacen que las empresas estén siendo eficientes en la selección de las estrategias, teniendo presente sus conocimientos,

${ }^{2}$ Facebook Live se diferencia de Facebook ya que es permite interacciones en vivo que facilitan que los clientes puedan expresar sus sentimientos y puntos de vista 

